\title{
Water Treadmill Training Ameliorates Neurite Outgrowth Inhibition Associated With NGR/RhoA/ROCK by Inhibiting Astrocyte Activation Following Spinal Cord Injury
}

\section{Xinwang Ying}

The Second Affiliated Hospital and Yuying Children's Hospital of Wenzhou Medical University

\section{Xiaolan Yu}

The Second Affiliated Hospital and Yuying Children's Hospital of Wenzhou Medical University Jintao Zhu

The Second Affiliated Hospital and Yuying Children's Hospital of Wenzhou Medical University

\section{Xuqing Li}

The Second Affiliated Hospital and Yuying Children's Hospital of Wenzhou Medical University

\section{Yujun Zheng}

The Second Affiliated Hospital and Yuying Children's Hospital of Wenzhou Medical University

\section{Qingfeng Xie}

The Second Affiliated Hospital and Yuying Children's Hospital of Wenzhou Medical University

\section{Qiaoyun Wu}

The Second Affiliated Hospital and Yuying Children's Hospital of Wenzhou Medical University

\section{Shengcun $\mathrm{Li}$}

The Second Affiliated Hospital and Yuying Children's Hospital of Wenzhou Medical University

\section{Jingjing Yue}

The Second Affiliated Hospital and Yuying Children's Hospital of Wenzhou Medical University

\section{Ye Zhou}

The First Affiliated Hospital of Zhejiang Chinese Medical University

\section{Kecheng Zhou}

The Second Affiliated Hospital and Yuying Children's Hospital of Wenzhou Medical University

Wenzhan Tu

The Second Affiliated Hospital and Yuying Children's Hospital of Wenzhou Medical University Songhe Jiang ( $\sim$ jiangsonghe@wmu.edu.cn )

The Second Affiliated Hospital and Yuying Children's Hospital of Wenzhou Medical University

\section{Research}


Keywords: Spinal cord injury, Water treadmill training, NGR/RhoA/ROCK, Neurite outgrowth, Astrocyte Posted Date: May 25th, 2021

DOI: https://doi.org/10.21203/rs.3.rs-532819/v1

License: (c) (1) This work is licensed under a Creative Commons Attribution 4.0 International License. Read Full License

Version of Record: A version of this preprint was published at Oxidative Medicine and Cellular Longevity on March 27th, 2022. See the published version at https://doi.org/10.1155/2022/1724362. 


\section{Abstract}

Background: Axons become scattered and incomplete after spinal cord injury (SCl). Cross-talk between astrocytes and neurons plays a pivotal role in neurite outgrowth following SCl. Rehabilitative training is a recognized method for the treatment of $\mathrm{SCl}$, but the specific mechanism of its effect on axonal outgrowth in the central nervous system (CNS) has not been determined.

Methods: A total of 160 adult male SD rats weighing $200-250 \mathrm{~g}$ were randomly divided into three groups, and an SCl animal model was established. Rats were subjected to water treadmill training (TT) for 7 or 14 d. The Basso-Beattie-Bresnahan (BBB) motor function scale, hematoxylin-eosin (HE) staining, Nissl staining, Western blotting and immunofluorescence were used to measure the degree of neurological deficit, tissue morphology, quantitative expression and accurate localization of the corresponding proteins.

Results: We found that TT decreased tissue structure damage and improved functional recovery. TT promoted the regeneration of neurons and reduced apoptosis induced by $\mathrm{SCl}$ around the lesion. TT significantly increased the expression of GAP43 and NF200 after SCI. In addition, the injury-induced increase in the expression of proinflammatory factors was significantly inhibited by TT. TT reduced the activation of astrocytes and microglia, accompanied by reduced expression of $\mathrm{C} 3 \mathrm{~d}$ and higher increased of S100A10. Finally, the level of chondroitin sulfate proteoglycan (CSPG) surrounding the lesion and activation of the NGR/RhoA/ROCK signalling pathway in neurons after SCl were effectively inhibited by TT.

Conclusions: In this study, we found that TT played a novel role in recovery from SCl by promoting axonal outgrowth associated with the NGR/RhoA/ROCK by inhibiting astrocyte activation after SCl.

\section{Introduction}

Axons become scattered and incomplete after spinal cord injury (SCI). The regenerative ability of damaged axons is poor, and regenerated axons fail to be guided well to form functional connections[1]. Therefore, finding a treatment that can promote axon regeneration and provide corresponding guiding signals so that regenerated axons can reform an effective conduction circuit and promote functional recovery is the main goal of rehabilitation after $\mathrm{SCl}$.

In the ischaemic and inflammatory microenvironment caused by SCl, the phenotype of naive astrocytes (NAs) changes, and the cells first become reactive astrocytes (RAs), and then scar-formed astrocytes $(\mathrm{SAs})[2,3]$. RAs can be divided into toxic $A 1$ astrocytes, which induce rapid death in neurons and oligodendrocytes, and neuroprotective A2 astrocytes, which promote neuronal survival and tissue repair[4]. RAs proliferate, migrate, and transform into SAs that form astrocytic scars around the lesion epicentre [5, 6]. One of the hallmark genes of RAs - chondroitin sulfate proteoglycan (CSPG) and glial scarring, is the core barriers against axonal regeneration in injured neurons caused by $\mathrm{SCl}[7,8]$. As such, 
elucidating the role of cross-talk between astrocytes and neurons in SCl may be helpful in developing new therapeutic strategies for SCl.

Nogo receptor 1 (NGR1) and NGR3 are two receptors of myelin-related inhibitors[9]. When these receptors interact with CSPG, they activate the Rho/ROCK signalling pathway and induce the collapse of axonal growth cones[10]. Rho family proteins are a group of guanosine triphosphate-binding proteins with relative molecular weights of approximately $20 \sim 30 \mathrm{kDa}$. The main function of Rho proteins is to regulate the recombination of the actin cytoskeleton through the downstream effector Rho kinase (ROCK) [11, 12]. It has been reported that there is almost no protein expression of RhoA/ROCK in mature neurons, but the expression of RhoA increases significantly after $\mathrm{SCl}$ (> 10 times)[13]. RhoA begins to appear as early as $1.5 \mathrm{~h}$ after injury and continues to be highly expressed for 4 weeks[9]. The in vivo $\mathrm{SCl}$ experiments conducted by Tomas $\mathrm{M}$ et al.[14] and Sabine $\mathrm{C}$ et al.[15] have reached a similar conclusion that there is a large amount of activated RhoA and ROCK expression in the processes of nerve cells in the injured area, and blocking these factors can promote axonal growth. On the other hand, the use of specific inhibitors of ROCK in vitro also indicated that ROCK had a negative regulatory effect on the activity of growth cones[16]. These studies show that the RhoA/ROCK pathway in neurons plays an indispensable role in the inhibition of axonal regeneration.

Rehabilitative training is currently one of the most effective nondrug treatments for $\mathrm{SCl}$, and the mechanism has not been verified. Studies have confirmed that rehabilitative training can increase the expression of central nervous system (CNS) neurotrophic factors, which is beneficial for corresponding nerve growth[17-19]. Surprisingly, Irin C. M et al reported that compulsory exercise therapy could promote the sprouting of axonal lateral branches[20]. It has also been reported that rehabilitative training can promote axonal regeneration in peripheral nerve injury[21], but the specific mechanism by which rehabilitative training affects axonal outgrowth in the CNS has not been determined. In this study, we combined a treadmill with swimming (avoiding possible injury caused by early intensive training) and used the water treadmill[22] to explore the further mechanism of neurite outgrowth after SCl.

\section{Materials And Methods}

\section{Antibodies}

5-bromo-20-deoxyuridine (BrdU), glial fibrillary acidic protein (GFAP), ionized calcium binding adapter molecule 1 (Iba1) and neurofilament-200(NF200) antibodies were purchased from Abcam (MC, UK). Ras homolog gene family, member A (RhoA), anti-C3d, anti-NGR, interleukin-1 $\beta$ (IL-1 $\beta$ ), interleukin-6 (IL-6), tumor necrosis factor a (TNFa), growth associated protein-43 (GAP43) and anti-GAPDH antibodies were purchased from Affinity (OH, USA). S100 calcium-binding protein A10 (S100A10), anti-ROCK2 were purchased from ABclonal Technology Co., Ltd (Wuhan, China). CSPGs was purchased from Merck Chemicals (Shanghai, China) Co., Ltd. Neuron-specific nuclear protein (NeuN) was purchased from Novus (Co, USA). Anti-Tubulin was purchased from Proteintech (IL, USA).

\section{Animals}


A total of 160 adult male SD rats weighing 200-250 g were obtained from Shanghai Laboratory Animal Centre. Rats were randomly divided into five groups: Sham-operated ( $n=40 ;$ group S); $7 \mathrm{~d}$ after SCl $(n=30$; group M7); $14 \mathrm{~d}$ after SCI ( $\mathrm{n}=30$; group M14); SCI + TT for $7 \mathrm{~d}$ ( $\mathrm{n=30}$; group TM7); SCI + TT for $14 \mathrm{~d}(\mathrm{n}=30$; group TM14). Rats are kept in a controlled environment and fed food and water regularly. All protocols were approved by the Animal Research Committee of Wenzhou Medical University.

\section{SCI Model}

Rats were kept anesthetized using $2 \%$ pentobarbital sodium $(30 \mathrm{mg} / \mathrm{kg})$. Then the T9-T11 area on the back of the rat was quickly scraped off, an incision of about $1 \mathrm{~cm}$ was made with a scalpel, and the lamina was peeled off with rongeurs to expose the corresponding spinal cord. A New York University Impactor $(10 \mathrm{~g} \times 20 \mathrm{~cm})$ was used to impact the exposed site except for group S. Spinal cord bruising, lower limb trembling and tail wagging indicate the success of the model. Finally, the wound is quickly sutured with absorbable surgical thread. In the following experimental phase, the bladder was emptied and the urethra was cleaned every morning and evening.

\section{Water treadmill training}

The rats will have $3 \mathrm{~d}$ of adaptive treadmill training before the establishment of the model. The setting of the treadmill (Wenzhou Xinglong Stainless Steel Co, LTD, Zhejiang, China): 10-15 m/min, $5 \mathrm{~min} /$ round, 3 rounds in total, 5 min interval between rounds, water temperature is maintained at $30^{\circ} \mathrm{C}$. The following day after $\mathrm{SCl}$, the rats in the TM group were treated with TT for $7 \mathrm{~d}$ or $14 \mathrm{~d}$, respectively. Each time before experiment, rats were put into water at $30{ }^{\circ} \mathrm{C}$ for defecation. After the experiment, the rats will be cleaned and dried and then put back into the cage.

\section{Behavioural tests}

Motor function was conducted by two independent examiners who were blinded to the treatment groups using the Basso-Beattie-Bresnahan (BBB) open-field test, which ranges from 0 (complete paralysis) to 21 (normal locomotion). The animals in each group $(n=10)$ were tested at $1,3,7$, and $14 \mathrm{~d}$ following injury.

\section{Tissue preparation}

Rats from each group were sacrificed at 7 and $14 \mathrm{~d}$ after $\mathrm{SCl}$. After $2 \%$ pentobarbital sodium deep anesthesia, rats were transcardially perfused with $0.9 \%$ sodium chloride at $4{ }^{\circ} \mathrm{C}$, followed by $4 \%$ paraformaldehyde in $0.1 \mathrm{M}$ phosphate buffer (PB, pH 7.4). For the rats to be stained with Brdu, $1 \%$ solution $(10 \mathrm{mg} / \mathrm{ml})$ was injected intraperitoneally 3 times every $4 \mathrm{~h}$ before the rats were sacrificed. Then the T9-T11 spinal cord tissue was removed from the rats quickly, stored in the same fixative for 24 hours $\left(4^{\circ} \mathrm{C}\right)$, and immersed in $0.1 \mathrm{M}$ phosphate-buffered $20 \%$ and $30 \%$ sucrose overnight at $4{ }^{\circ} \mathrm{C}$, respectively. Successive sections (15- $\mu \mathrm{m}$ thick) were frozen and stored for subsequent experiments.

\section{Haematoxylin-eosin (HE) and Nissl staining}


Prepared sections ( $n=5$ per group) were naturally air-dried for $3 \mathrm{~min}$. Then sections were stained with haematoxylin and eosin for HE staining and cresyl violet for Nissl staining, respectively. Finally, the Olympus BH- 2 microscope (Olympus Optics, London, UK) was used to capture the image and the size of cavity area was quantitatively counted.

\section{TUNEL staining}

Prepared sections ( $n=5$ per group) were collected for TUNEL staining. Permeabilized the slides for 10 min at room temperature and washed the slides three times for $15 \mathrm{~min}$. Then an In Situ Cell Death Detection Kit (Roche Molecular Biochemicals) was used to detect the apoptotic cells. Using a microscope (BX51, Olympus) to observe the images. The apoptosis positive cells were counted quantitatively using ImagePro Plus 6.0 analysis software.

\section{RT-PCR}

The primer sequences were 5'TGAGGAGAAGAAGGGCGAAGGG 3' (Forward), 5'AGGACGGCGAGTTATCAGTGGTAG3' (Reverse) for GAP43, 5'ACGCTGCAGTCAGAGGAGTG3' (Forward), 5'CTGCCGCCGGTACTCAGTTA 3' (Reverse) for NF200. High Capacity RNA-to-cDNA Master Mix (A3500, Promega) was applied to reverse transcribed the one microgram of total RNA. Real-time amplification was performed using SYBR Green (QPK-212, Tokyo, Japan) and a Light Cycler480 (Roche, USA). To amplify genomic DNA, PCR was performed under the following conditions: reverse transcription at $50{ }^{\circ} \mathrm{C}$ for 3 min, DNA polymerase activation and RT enzyme inactivation at $95^{\circ} \mathrm{C}$ for 5 min, followed by 45 cycles of denaturation at $95^{\circ} \mathrm{C}$ for $10 \mathrm{~s}$, primer annealing at $60^{\circ} \mathrm{C}$ for $10 \mathrm{~s}$, and elongation at $72{ }^{\circ} \mathrm{C}$ for 10 s. Comparative mRNA expression levels were expressed as $2^{-\Delta \Delta C t}$.

\section{Western blot Analysis}

At 7 and $14 \mathrm{~d}$ after injury, rats ( $\mathrm{n}=5$ each group) were sacrificed and a 5-mm length of T10 spinal cord was obtained. After the tissue is fully homogenized, the supernatant is taken. Then the extracted protein concentration was quantified by the bicinchoninic acid (BCA) assay kit. A separation gel suitable for the molecular weight of the protein was prepared for electrophoresis, and separated proteins were transferred to a PVDF membrane (Bio-Rad, Hercules, CA, USA). After a 90-minute 5\% milk closure, the membrane was washed three times with Tris-buffered saline solution with Tween (TBST)and incubated with the corresponding primary antibodies at $4{ }^{\circ} \mathrm{C}$ for 16-24 h: GAP43 (1:1000), NF200 (1:1000), IL-1ß (1:500), IL-6 (1:1500), TNFa (1:500), GFAP (1:1000), Iba1 (1:1000), C3d (1:500), S100A10 (1:500), CSPGs (1:500), NGR (1:500), RhoA (1:1000), ROCK2 (1:500), Tubulin (1:1000), GAPDH (1:1000). The secondary antibodies were incubated for $2 \mathrm{~h}$ and washed with TBST 3 times. All experiments were repeated three times. Images were visualized by the ChemiDicTM XRS + Imaging System (Bio-Rad).

\section{Immunofluorescence Staining}


Prepared sections were naturally air-dried and washed three times in $0.01 \mathrm{M}$ PBS, blocked with $10 \%$ normal goat serum at room temperature for $1 \mathrm{~h}$, and incubated with rabbit anti-GAP43 (1:200), mouse anti-NF200 (1:200), mouse anti-Brdu (1:200), goat (rabbit) anti-NeuN (1:100), mouse (rabbit) anti-GFAP (1:500), rabbit anti-lba1(1:300), rabbit anti-C3d (1:200), rabbit anti-S100A10 (1:20), mouse anti-CSPGs (1:200), rabbit anti-ROCK2 (1:100), rabbit anti-RhoA (1:200) and rabbit anti-NGR (1:200) overnight at $4{ }^{\circ} \mathrm{C}$. The second day, the sections were incubated with secondary antibodies: cy3-conjugated goat anti-rabbit (1:300), Alexa Flour 488-conjugated goat anti-mouse (1:400), cy3-conjugated goat anti-mouse (1:300), Alexa Flour 488-conjugated goat anti-rabbit (1:400) at room temperature for $50 \mathrm{~min}$. Nuclei were counterstained with 4,6-diamidino-2-phenylindole (DAPI). The fluorescence signal was observed under laser confocal microscopy. Five fields on each of three slides per animal were randomly selected for visualization and analysis performed using ImageJ software (National Institutes of Health, Bethesda, MD, USA).

\section{Statistical Analysis}

All experimental values are presented as the mean \pm SD. The Kolmogorov-Smirnov

test was used as a normality test, with $p>0.05$ indicating a normal distribution. Levene's test was used as a test of homogeneity of variance, with $p>0.05$ used to indicate homogeneous variance, and vice versa. When there were two experimental groups, Student's t-test was used. Statistical differences among groups were analyzed using two-way ANOVA followed by Tukey's test. BBB scores were detected using repeated measurement two-way ANOVA with group and time as factors, followed by Tukey's test to detect differences between groups. SPSS 16 statistical software was used for statistical analysis, and $p<0.05$ was considered statistically significant.

\section{Results}

\section{TT decreased tissue structure damage and improved functional recovery after $\mathrm{SCl}$}

At 7 and $14 \mathrm{~d}$ after injury, HE staining was used to observe the morphological and histological differences in the T9-T11 spinal cord (Fig. 1A). The tissue in the $S$ group was arranged closely, but the structural integrity of tissue in the $\mathrm{M}$ group was obviously destroyed, and the injured tissue formed some pathological holes after digestion and absorption. Quantitative analysis of the cavity area at $14 \mathrm{~d}$ showed that the cavity area in the TM group was significantly smaller than that in the $\mathrm{M}$ group after TT (Fig. 1B) (TM vs. M: $p<0.001$ ). Functional recovery was evaluated by BBB motor functional scoring at $1 \mathrm{~d}, 3 \mathrm{~d}, 7 \mathrm{~d}$, and $14 \mathrm{~d}$ after $\mathrm{SCl}$. According to the experimental results (Fig. 1C), the scores in the TM group were significantly higher than those in the M group at 7 and $14 \mathrm{~d}$ (TM vs. M: $7 \mathrm{~d}, p<0.01 ; 14 \mathrm{~d}, p<0.001$ ). These results indicate that TT can significantly improve functional recovery and preserve tissue.

\section{TT promoted the regeneration of neurons and reduced apoptosis around the lesion}


To observe neuronal damage, rostral to caudal Nissl staining was performed at $7 \mathrm{~d}$ in the different groups (Fig. 2A-B). As shown in Fig. 2C, compared with that in the $\mathrm{S}$ group, the number of $\mathrm{Niss}^{+}$cells in the $\mathrm{M}$ group decreased significantly in the rostral/epicentre/caudal area (M vs. S: rostral, $p<0.001$; epicentre, $p$ $<0.001$; caudal, $p<0.01)$. However, the number of Niss ${ }^{+}$cells increased significantly in the TM group (TM vs. M: rostral, $p<0.001$; epicentre, $p<0.01$; caudal, $p<0.01$ ). We further measured apoptosis (TUNEL staining) in each group $7 \mathrm{~d}$ after $\mathrm{SCl}$ (Fig. 2E). The number of apoptotic cells increased significantly after $\mathrm{SCl}$ (M vs. S: rostral/epicentre/caudal, $p<0.001$ ), and compared with that in the $\mathrm{M}$ group, the number of TUNEL ${ }^{+}$cells in the TM group was significantly decreased (TM vs. M: rostral/ epicentre /caudal, $p<$ 0.001) (Fig. 2G). At $14 \mathrm{~d}$ after injury, we used the neuronal marker NeuN and the regeneration marker BrdU to observe neuronal regeneration (Fig. 2D). As shown in Fig. 2F, nerve regeneration $\left(\mathrm{NeuN}^{+} / \mathrm{BrdU}^{+} \mathrm{co}-\right.$ labelled cells) in the TM group was increased compared to that with M group (TM vs. M: $p<0.01$ ).

\section{TT promoted axonal outgrowth after SCI}

Staining for GAP43/NF200 was used to determine the effects of TT on axonal outgrowth around the epicentre at $14 \mathrm{~d}$ after SCI (Fig. 3A-C). In the penumbra (1-3 mm adjacent to the epicentre), we found that the positive area of $\mathrm{GAP}_{4} 3^{+}$cells decreased significantly after $\mathrm{SCl}$ (Fig. 3D). In contrast, there was more GAP43 expression in the TM group than in the M group (M vs. S: $p<0.01 ;$ TM vs. M: $p<0.05$ ). Moreover, the protein expression of GAP43 and NF200 in each group was also measured by Western blotting (Fig. 3E). According to the results (Fig. 3F-G), GAP43 and NF200 protein expression was decreased to some extent after $\mathrm{SCl}$ at $7 \mathrm{~d}$ and $14 \mathrm{~d}$ compared to the levels in the $\mathrm{S}$ group, and the levels in the TT group were between those of the other two groups (GAP43, M vs. S: $p<0.001, \mathrm{TM}_{7}$ vs. $\mathrm{M}_{7}: p<0.01, \mathrm{TM}_{14}$ vs. $\mathrm{M}_{14}$ : $p<$ $0.001 ;$ NF200, $M_{7}$ vs. $S: p<0.001, \mathrm{TM}_{7}$ vs. $\mathrm{M}_{7}: p<0.001, \mathrm{TM}_{14}$ vs. $\left.\mathrm{M}_{14}: p<0.001\right)$. RT-qPCR analysis was performed (Fig. $3 \mathrm{H}-\mathrm{I})$, and there were significant differences in GAP43 and NF200 mRNA expression levels between the $\mathrm{M}$ and TM groups (GAP43 mRNA, $\mathrm{M}_{7}$ vs. $\mathrm{S}: p<0.001, \mathrm{TM}_{7}$ vs. $\mathrm{M}_{7}: p<0.01, \mathrm{TM}_{14}$ vs. $\mathrm{M}_{14}$ : $p<$ 0.05 ; NF200 mRNA, $M_{7}$ vs. $S: p<0.001, \mathrm{TM}_{7}$ vs. $\mathrm{M}_{7}: p<0.05, \mathrm{TM}_{14}$ vs. $\left.\mathrm{M}_{14}: p<0.05\right)$.

\section{TT inhibited the inflammatory activation of microglia and astrocytes}

In the T9-T11 spinal cord segment, the total protein expression of proinflammatory factors (IL-1 $\beta$, IL-6, and TNF-a) and glial cells (GFAP and Iba1) protein were measured by Western blotting (Fig. 4A-B). We normalized the protein levels and then analysed the data in comparison with the corresponding group (IL$1 \beta, M_{7}$ vs. $S: p<0.001, \mathrm{TM}_{7}$ vs. $\mathrm{M}_{7}: p<0.001, \mathrm{TM}_{14}$ vs. $\mathrm{M}_{14}: p<0.001 ; \mathrm{IL}-6, \mathrm{M}_{7}$ vs. $\mathrm{S}: p<0.001, \mathrm{TM}_{7}$ vs. $\mathrm{M}_{7}$ : $p<0.01, \mathrm{TM}_{14}$ vs. $\mathrm{M}_{14}: p<0.05 ;$ TNF-a, $\mathrm{M}_{7}$ vs. $\mathrm{S}: p<0.001, \mathrm{TM}_{7}$ vs. $\mathrm{M}_{7}: p<0.05, \mathrm{TM}_{14}$ vs. $\mathrm{M}_{14}: p<0.05$; GFAP, $M_{7}$ vs. $S: p<0.001, \mathrm{TM}_{7}$ vs. $\mathrm{M}_{7}: p<0.01, \mathrm{TM}_{14}$ vs. $\mathrm{M}_{14}: p<0.001$; Iba1, $\mathrm{M}_{7}$ vs. $\mathrm{S}: p<0.001, \mathrm{TM}_{7}$ vs. $\mathrm{M}_{7}: p<0.05, \mathrm{TM}_{14}$ vs. $\mathrm{M}_{14}: p<0.01$ ) (Fig. 4 C). To examine astrocyte proliferation, we used Ki67 to fluorescently label proliferating cells and GFAP to fluorescently label astrocytes and analysed the degree of co-staining (Fig. 4D). The number of $\mathrm{GFAP}^{+} / \mathrm{Ki} 7^{+}$cells significantly increased at $7 \mathrm{~d}$ after injury, and this outcome was improved by TT (M vs. S: $p<0.01$; TM vs. M: $p<0.01$ ) (Fig. 4E). In addition, we also performed GFAP/Iba1 co-staining experiments to observe the activation and positional connection of 
glial cells (Fig. 4F). As shown in Fig. 4G, astrocytes and microglia were activated, and TT the number of activated glial cells decreased significantly after SCI (GFAP-expressing astrocytes, M vs. S: $p<0.001, \mathrm{TM}$ vs. M: $p<0.001$; activated microglial microglia intensity, M vs. S: $p<0.01$, TM vs. M: $p<0.01$ ).

\section{TT inhibited A1-type reactive astrocytes and promoted A2-type reactive astrocytes around the lesion}

In the penumbra, the protein expression levels of the A1 type astrocyte marker C3d and the A2 type astrocyte marker S100A10 were measured by Western blotting (Fig. 5A). Compared with that in the S group, the protein expression of C3d was markedly increased in the M group (M vs. S: $p<0.001$ ), and this effect was reduced by TT (TM vs. M: $7 d, p<0.01 ; 14 d, p<0.001$ ). Additionally, the S100A10 protein level was increased in the M group ( $M$ vs. S: $p<0.01$ ), accompanied by a higher expression in the TM group (TM vs. M: 7 d, $p<0.001 ; 14$ d, $p<0.05$ ) (Fig. 5B). We further performed co-staining of GFAP/C3d and GFAP/S100A10 (Fig. 5E-F). As shown in Fig. 5C, the results indicated that C3d expression in astrocytes increased significantly after injury but decreased after TT (M vs. S: $p<0.01 ;$ TM vs. M: $p<0.01$ ). In contrast to the trend in the protein expression of C3d, the protein expression of S100A10 in astrocytes increased after $\mathrm{SCl}$ and further increased after TT intervention (M vs. S: $p<0.001 ; \mathrm{TM}$ vs. M: $p<0.01$ ) (Fig. 5D). These results suggest that after TT, A1 astrocyte activation is inhibited to a certain extent, while A2 astrocyte activation is promoted.

\section{TT inhibited the expression of RhoA/Rock induced by astrocyte activation}

To study the cross-talk between astrocytes and neurons, CSPG and NGR/RhoA/ROCK signalling pathway protein expression in the penumbra was measured by Western blotting at 7 and $14 \mathrm{~d}$ (Fig. 6A). As shown in Fig. 6B, after injury, the expression of CSPG increased significantly, while the expression of CSPG in the TT group decreased significantly compared with that in the $\mathrm{M}$ group, and the effect of TT became more obvious at $14 \mathrm{~d}\left(\mathrm{M}_{7}\right.$ vs. S: $p<0.001, \mathrm{TM}_{7}$ vs. $\mathrm{M}_{7}: p<0.05, \mathrm{TM}_{14}$ vs. $\left.\mathrm{M}_{14}: p<0.001\right)$. We found that the expression of the NGR/RhoA/ROCK signalling pathway was strikingly similar to the trend in CSPG expression (NGR, $M_{7}$ vs. $S: p<0.001, \mathrm{TM}_{7}$ vs. $\mathrm{M}_{7}: p<0.01$, $\mathrm{TM}_{14}$ vs. $\mathrm{M}_{14}: p<0.01$; RhoA, $\mathrm{M}_{7}$ vs. $\mathrm{S}: p<$ $0.001, \mathrm{TM}_{7}$ vs. $\mathrm{M}_{7}: p<0.01, \mathrm{TM}_{14}$ vs. $\mathrm{M}_{14}: p<0.001 ; \mathrm{ROCK} 2, \mathrm{M}_{7}$ vs. $\mathrm{S}: p<0.001, \mathrm{TM}_{7}$ vs. $\mathrm{M}_{7}: p<0.05, \mathrm{TM}_{14}$ vs. $\left.M_{14}: p<0.05\right)$. To further determine the localized expression of CSPG, we co-stained the tissue for GFAP and CSPG (Fig. 6B). Consistent with the previous trend in protein expression, astrocytes produced CSPG around the epicentre after $\mathrm{SCl}$, and this effect was significantly improved in the TT group. We also carried out localized multilabel staining of NGR/RhoA/ROCK signalling pathway factors at $14 \mathrm{~d}$ to further verify the experimental results. As shown in Fig. 6D-E, the expression of RhoA/ROCK in neurons increased significantly after $\mathrm{SCl}$ but decreased after TT. Ultimately, we performed triple-staining for NeuN/NGR/CSPG to observe the binding of CSPG produced by astrocytes to NGR on the membranes of neurons (Fig. 7). CSPG binds to NGR on neurons after injury, thus activating downstream signalling pathways. Compared with that in the $\mathrm{M}$ group, the number of co-labelled cells in the TM group decreased.

\section{Discussion}


General drug intervention hardly promotes effective growth in retracted axons to form functional connections, which has always been a challenge in $\mathrm{SCl}$ treatment. As a non-invasive and effective treatment, rehabilitation training has been gradually accepted and widely used in the clinic, but the specific mechanism of its effect on $\mathrm{SCl}$ has not been fully elucidated. In this study, we found that water treadmill training played a novel role in recovery from SCl by promoting axonal outgrowth associated with NGR/RhoA/ROCK by inhibiting astrocyte activation.

Early rehabilitation exercise after $\mathrm{SCl}$ can maintain muscle strength and prevent joint contracture[23, 24]. Previous studies have shown that the expression of neurotrophin increases after a certain intensity of TT in rats with $\mathrm{SCI}[17,25]$. Furthermore, TT can also attenuate blood-spinal cord barrier disruption[22], which provides nutrition for nerve cells. Focusing on paralysis of the lower limbs after injury in rats, we combined swimming with a treadmill to allow rats to perform active rehabilitation training through the synergistic effect of water flow and the conveyor belt. The current experimental results show that the motor function of rats was significantly improved after treadmill training for $14 \mathrm{~d}$. In addition, GAP43 and NF200 were used to evaluate neuronal and axonal injury[26-28]. We found that the expression of GAP43 and NF200 was significantly decreased due to neuronal injury. We were surprised to find that after $14 \mathrm{~d}$ of training, the expression of GAP43 and NF200 in the penumbra increased significantly, accompanied by an increase in the number of regenerated neurons, indicating the neuroprotective effect of TT. To further explore the underlying mechanism of TT, we first focused on the signalling molecules in neurons.

The Nogo receptor family members NgR1 and NgR3 bind to CSPG, activating a signalling cascade via Rho family GTPases $[9,29,30]$. RhoA is the most important Rho family gene associated with nerve regeneration[7]. The main signalling molecule downstream of RhoA is Ras homologue-associated kinase [31]. RhoA/ROCK activation, in turn, activates downstream effectors, which regulate cytoskeletal reorganization such as growth cone collapse and neurite outgrowth inhibition[32]. Studies have shown that inhibiting RhoA/ROCK improves neurite outgrowth[14, 15]. Furthermore, inhibiting ROCK with Y27632 promotes axonal regeneration in SCI $[11,16]$. Interestingly, we found that the expression of the RhoA/ROCK signalling pathway in neurons increased significantly after $\mathrm{SCl}$, but decreased significantly after TT (Fig.6). As expected, the experimental results suggest that the neuroprotective effect of TT after $\mathrm{SCl}$ is closely associated with the RhoA/ROCK signalling pathway. Therefore, we did additional in-depth research.

Glial cells, which are the most abundant cell type in the CNS, play vital roles in maintaining CNS homeostasis [5]. Astrocytes exhibit different morphological and functional changes after nociceptive stimulation, including (1) morphological changes, (2) proliferation, (3) changes in gene expression, (4) significant molecular changes, and (5) functional changes[6]. Immediately after SCl, oxidative stress causes microglial and astrocyte activation, and these cells release excessive amounts of proinflammatory mediators, such as IL- $1 \beta, \mathrm{IL}-6$, and TNF- $\alpha$, which are directly deleterious to neighbouring neurons $[33,34]$. As expected, in our experiment, we found that the abundant expression of proinflammatory factors (IL-1 $\beta$, IL-6, and TNF-a) induced by SCl decreased significantly after TT (Fig.4). The fluorescence staining results showed that astrocytes were significantly hypertrophic following $\mathrm{SCl}$, 
and the Ki67/GFAP co-staining results showed a large number of astrocytes proliferating after SCl. Furthermore, our study also suggests that injury induces the activation of microglia, and by comparing the results of GFAP/lba1 co-staining between the S group and M group, we found that these two cell types close to each other after activation, indicating that there may be coactivation between these cells. In contrast, this phenomenon was alleviated in the TM group, indicating that TT can significantly inhibit the activation of astrocytes and microglia.

After noxious stimulation and nerve injury, the phenotype, functions, and gene expression of astrocytes can undergo significant changes known as reactive astrogliosis[5]. Consistent with the results of the latest stroke study, we found that $\mathrm{SCl}$ could induce the activation of two kinds of astrocytes: neurotoxic A1 astrocytes release neurotoxic complement C3d, resulting in neuronal death, while A2 astrocytes release S100A10 to protect neurons[4]. The dual roles of reactive astrocytes after SCI have prompted further thought. Is it possible that the transformation of $A 1$ and $A 2$ reactive astrocytes is a strategy to prevent and treat $\mathrm{SCl}$ ? As we hypothesized, the expression of the A1- and A2-type marker proteins $\mathrm{C} 3 \mathrm{~d}$ and S100A10, respectively, was significantly upregulated around the lesion after SCl. However, after TT, the number of A1 RAs decreased significantly, while A2 RAs were increased, suggesting that TT can inhibit A1-type RAs and promote A2-type RAs (Fig. 5).

Thereafter, RAs proliferate, migrate, and transform into $S A s[2,3,35]$. The most representative marker gene of SAs is CSPG, which binds to NGR1 and NGR3 on neurons and activates the downstream RhoA/ROCK signalling pathway to inhibit neurite growth $[6,36]$. In view of previous studies, we examined the expression of CSPG and its localization and binding in neurons after $\mathrm{SCl}$. We found that $7 \mathrm{~d}$ after injury, a large amount of CSPG was secreted by astrocytes around the injury site, but the level decreased significantly in the TT group. The expression of CSPG was downregulated $14 \mathrm{~d}$ after injury, especially in the TM group (Fig.6). In addition, the number of $\mathrm{NeuN}^{+} / \mathrm{NGR}^{+} / \mathrm{CSPG}^{+}$cells in the $\mathrm{M}$ group was significantly higher than that in the S group, indicating that a large amount of CSPG in the extracellular matrix bound to NGR on neurons after injury. We were surprised to find that the number of co-labelled cells in the TM group was much lower than that in the M group (Fig.7). The therapeutic effect of TT may be associated with the decrease in the number of activated SAs, which also explains the root cause of the decrease in RhoA/ROCK activation in neurons in the TM group. In summary, these results suggest that TT can effectively promote axonal growth after $\mathrm{SCl}$, partly by regulating RA activation to reduce activation of the RhoA/ROCK signalling pathway in neurons.

However, the major limitation of this study should be noted: we only used male rats, ignoring any possible differences caused by sex. To our knowledge, there is no effective treatment for $\mathrm{SCl}$ that can promote the growth of injured axons without any side effects. Fortunately, our team has been working on developing a motor therapy that includes early treatment intervention to promote axonal growth and elucidate the underlying mechanism, and the aim is to translate this method into a clinical treatment.

\section{Conclusion}


In conclusion, our study presented proof that TT ameliorates neurite outgrowth inhibition. Additionally, TT decreased the activation of astrocytes after $\mathrm{SCl}$, decreased the expression of $\mathrm{A} 1$ astrocytes and increased the expression of A2 astrocytes. Ultimately, TT reduced the CSPGs, secreted by SAs, thus reducing the activation of RhoA/ROCK signaling pathway, which indirectly reduced the inhibition of neurite outgrowth(Fig. 8).

\section{Abbreviations}

SCI: spinal cord injury; RAs: reactive astrocytes; SAs: scar-formed astrocytes; CSPGs: chondroitin sulfate proteoglycan; NGR: nogo receptor; h: hour; d: day; CNS: central nervous system; Brdu: 5-bromo-20deoxyuridine; GFAP: glial fibrillary acidic protein; Iba1: ionized calcium binding adapter molecule 1; NF200: neurofilament-200; RhoA: ras homolog gene family, member A; IL-1ß: interleukin-1 $\beta$; IL-6: interleukin-6; TNFa: tumor necrosis factor a; GAP43: growth associated protein-43; S100A10: S100 calcium-binding protein A10; CSPGs: chondroitin sulfate proteoglycans; NeuN: neuron-specific nuclear protein; TT: treadmill training; BBB: Basso-Beattie-Bresnahan; HE: haematoxylin-eosin; BCA: bicinchoninic acid; TBS: tris-buffered saline solution; DAPI: 4,6-diamidino-2-phenylindole.

\section{Declarations}

\section{Acknowledgements}

Not applicable

\section{Authors' contributions}

YXW and YXL conducted most of the experiments and drafted the manuscript. ZKC and LSC participated in the design of the experiment; XQF and ZJT were responsible for data acquisition and analysis; LXQ, ZYJ, YJJ and WQY conceived the experiments; TWZ and ZY provided guidance; JSH supervised the whole process of the experiment, and was a major contributor in writing the manuscript. All authors read and approved the final manuscript.

\section{Funding}

This research was supported by the Zhejiang Provincial Natural Science Foundation of China (No. LQ21H170003) and National Natural Science Foundation of China (No.81873376). We would like to express our heartfelt thanks to all the technical personnel who have provided assistance in this work.

\section{Availability of data and materials}

The datasets supporting the conclusions of this article are available from the corresponding author on reasonable request.

\section{Ethics approval and consent to participate}


The protocols were approved by the Animal Research Committee of Wenzhou Medical University. The maintenance and handling of the rats were consistent with the guidelines of the National Institutes of Health, and adequate measures were taken to minimize animal discomfort.

\section{Consent for publication}

Not applicable.

\section{Competing interests}

The authors declare that they have no competing interests.

\section{Author details}

${ }^{a}$ Department of Physical Medicine and Rehabilitation, The Second Affiliated Hospital and Yuying Children's Hospital of Wenzhou Medical University, Zhejiang, Wenzhou 325000, China. ${ }^{b}$ Department of Intelligent Rehabilitation International (Cross-Strait), Alliance of Wenzhou Medical University, Zhejiang, Wenzhou 325000, China. ${ }^{C}$ The First Affiliated Hospital of Zhejiang Chinese Medical University (Zhejiang Provincial Hospital of Traditional Chinese Medicine)

\section{References}

1. Fouad K, Tetzlaff W. Rehabilitative training and plasticity following spinal cord injury. Exp Neurol. 2012;235:91-9.

2. Liddelow SA, Barres BA. Reactive Astrocytes: Production, Function, and Therapeutic Potential. Immunity. 2017;46:957-67.

3. Okada S, Hara M, Kobayakawa K, Matsumoto Y, Nakashima Y. Astrocyte reactivity and astrogliosis after spinal cord injury. Neurosci Res. 2018;126:39-43.

4. Liddelow SA, Guttenplan KA, Clarke LE, Bennett FC, Bohlen CJ, Schirmer L, Bennett ML, Münch AE, Chung WS, Peterson TC, et al. Neurotoxic reactive astrocytes are induced by activated microglia. Nature. 2017;541:481-7.

5. Hara M, Kobayakawa K. Interaction of reactive astrocytes with type I collagen induces astrocytic scar formation through the integrin-N-cadherin pathway after spinal cord injury. 2017, 23:818-828.

6. Li T, Chen X, Zhang C, Zhang Y, Yao W. An update on reactive astrocytes in chronic pain. J Neuroinflammation. 2019;16:140.

7. Tep C, Lim TH, Ko PO, Getahun S, Ryu JC, Goettl VM, Massa SM, Basso M, Longo FM, Yoon SO. Oral administration of a small molecule targeted to block proNGF binding to $\mathrm{p} 75$ promotes myelin sparing and functional recovery after spinal cord injury. J Neurosci. 2013;33:397-410.

8. Vadivelu S, Stewart TJ, Qu Y, Horn K, Liu S, Li Q, Silver J, McDonald JW. NG2 + progenitors derived from embryonic stem cells penetrate glial scar and promote axonal outgrowth into white matter after spinal cord injury. Stem Cells Transl Med. 2015;4:401-11. 
9. Dickendesher TL, Baldwin KT, Mironova YA, Koriyama Y, Raiker SJ, Askew KL, Wood A, Geoffroy CG, Zheng B, Liepmann CD, et al. NgR1 and NgR3 are receptors for chondroitin sulfate proteoglycans. Nat Neurosci. 2012;15:703-12.

10. Sami A, Selzer ME, Li S. Advances in the Signaling Pathways Downstream of Glial-Scar Axon Growth Inhibitors. Front Cell Neurosci. 2020;14:174.

11. Peng Z, Li X, Fu M, Zhu K, Long L, Zhao X, Chen Q, Deng DYB, Wan Y. Inhibition of Notch1 signaling promotes neuronal differentiation and improves functional recovery in spinal cord injury through suppressing the activation of Ras homolog family member A. 2019, 150:709-722.

12. Stankiewicz TR, Linseman DA. Rho family GTPases: key players in neuronal development, neuronal survival, and neurodegeneration. Front Cell Neurosci. 2014;8:314.

13. Gutekunst CA, Tung JK, McDougal ME, Gross RE. C3 transferase gene therapy for continuous conditional RhoA inhibition. Neuroscience. 2016;339:308-18.

14. Madura T, Yamashita T, Kubo T, Fujitani M, Hosokawa K, Tohyama M. Activation of Rho in the injured axons following spinal cord injury. EMBO Rep. 2004;5:412-7.

15. Conrad S, Schluesener HJ, Trautmann K, Joannin N, Meyermann R, Schwab JM. Prolonged lesional expression of RhoA and RhoB following spinal cord injury. J Comp Neurol. 2005;487:166-75.

16. Hirose M, Ishizaki T, Watanabe N, Uehata M, Kranenburg O, Moolenaar WH, Matsumura F, Maekawa $\mathrm{M}$, Bito H, Narumiya S. Molecular dissection of the Rho-associated protein kinase (p160ROCK)regulated neurite remodeling in neuroblastoma N1E-115 cells. J Cell Biol. 1998;141:1625-36.

17. Sandrow-Feinberg HR, Houlé JD. Exercise after spinal cord injury as an agent for neuroprotection, regeneration and rehabilitation. Brain Res. 2015;1619:12-21.

18. Ying X, Tu W, Li S, Wu Q, Chen X, Zhou Y, Hu J, Yang G, Jiang S. Hyperbaric oxygen therapy reduces apoptosis and dendritic/synaptic degeneration via the BDNF/TrkB signaling pathways in $\mathrm{SCl}$ rats. Life Sci. 2019;229:187-99.

19. Ying X, Xie Q, Yu X, Li S, Wu Q, Chen X, Yue J, Zhou K, Tu W, Jiang S. Water treadmill training protects the integrity of the blood-spinal cord barrier following SCI via the BDNF/TrkB-CREB signalling pathway. Neurochem Int. 2021;143:104945.

20. Maier IC, Baumann K, Thallmair M, Weinmann O, Scholl J, Schwab ME. Constraint-induced movement therapy in the adult rat after unilateral corticospinal tract injury. J Neurosci. 2008;28:9386-403.

21. Sachdeva R, Theisen CC, Ninan V, Twiss JL, Houlé JD. Exercise dependent increase in axon regeneration into peripheral nerve grafts by propriospinal but not sensory neurons after spinal cord injury is associated with modulation of regeneration-associated genes. Exp Neurol. 2016;276:72-82.

22. Ying X, Xie Q, Li S, Yu X, Zhou K, Yue J, Chen X, Tu W, Yang G, Jiang S. Water treadmill training attenuates blood-spinal cord barrier disruption in rats by promoting angiogenesis and inhibiting matrix metalloproteinase-2/9 expression following spinal cord injury. Fluids Barriers CNS. 2020;17:70. 
23. Hughes DC, Ellefsen S, Baar K. Adaptations to Endurance and Strength Training. Cold Spring Harb Perspect Med 2018, 8.

24. Wang R, Tian H, Guo D, Tian Q, Yao T, Kong X. Impacts of exercise intervention on various diseases in rats. J Sport Health Sci. 2020;9:211-27.

25. Li X, Wu Q, Xie C, Wang C, Wang Q, Dong C, Fang L, Ding J, Wang T. Blocking of BDNF-TrkB signaling inhibits the promotion effect of neurological function recovery after treadmill training in rats with spinal cord injury. Spinal Cord. 2019;57:65-74.

26. Zhu S, Ying Y, Ye J, Chen M, Wu Q, Dou H, Ni W, Xu H, Xu J. AAV2-mediated and hypoxia response element-directed expression of bFGF in neural stem cells showed therapeutic effects on spinal cord injury in rats. 2021, 12:274.

27. Zhu S, Ying Y, Ye L, Ying W, Ye J, Wu Q, Chen M, Zhu H, Li X, Dou H, et al. Systemic Administration of Fibroblast Growth Factor 21 Improves the Recovery of Spinal Cord Injury (SCI) in Rats and Attenuates SCI-Induced Autophagy. Front Pharmacol. 2020;11:628369.

28. Zhu J, Lu Y, Yu F, Zhou L, Shi J, Chen Q, Ding W, Wen X, Ding YQ, Mei J, Wang J. Effect of decellularized spinal scaffolds on spinal axon regeneration in rats. J Biomed Mater Res A. 2018;106:698-705.

29. Schwab ME. Functions of Nogo proteins and their receptors in the nervous system. Nat Rev Neurosci. 2010;11:799-811.

30. Mohammed R, Opara K, Lall R, Ojha U, Xiang J. Evaluating the effectiveness of anti-Nogo treatment in spinal cord injuries. Neural Dev. 2020;15:1.

31. Jennings RT, Knaus UG. Rho family and Rap GTPase activation assays. Methods Mol Biol. 2014;1124:79-88.

32. Fujita Y, Yamashita T. Axon growth inhibition by RhoA/ROCK in the central nervous system. Front Neurosci. 2014;8:338.

33. Wang L, Yao Y, He R, Meng Y, Li N, Zhang D, Xu J, Chen O, Cui J, Bian J, et al. Methane ameliorates spinal cord ischemia-reperfusion injury in rats: Antioxidant, anti-inflammatory and anti-apoptotic activity mediated by Nrf2 activation. Free Radic Biol Med. 2017;103:69-86.

34. Liu M, Xu Z, Wang L, Zhang L, Liu Y, Cao J, Fu Q, Liu Y, Li H, Lou J, et al: Cottonseed oil alleviates ischemic stroke injury by inhibiting the inflammatory activation of microglia and astrocyte. 2020, 17:270.

35. Franco Rodríguez NE, Dueñas Jiménez JM, De la Torre Valdovinos B, López Ruiz JR. Hernández Hernández L, Dueñas Jiménez SH: Tamoxifen favoured the rat sensorial cortex regeneration after a penetrating brain injury. Brain Res Bull. 2013;98:64-75.

36. Silver J, Miller JH. Regeneration beyond the glial scar. Nat Rev Neurosci. 2004;5:146-56.

\section{Figures}


A

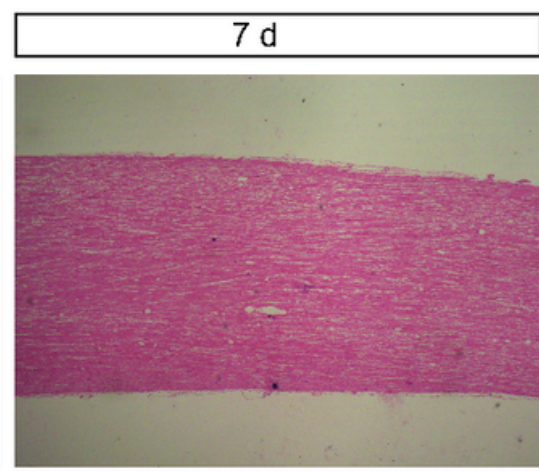

$\sum$
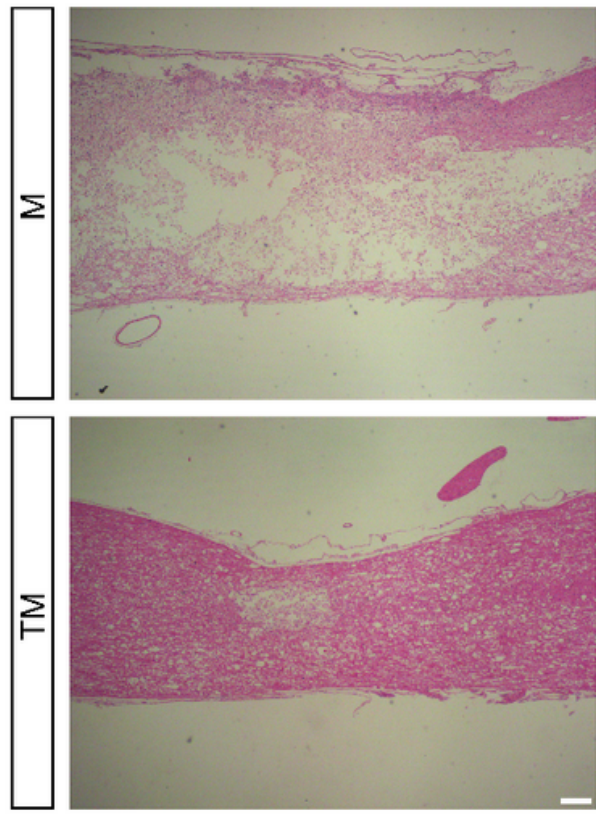

$14 \mathrm{~d}$
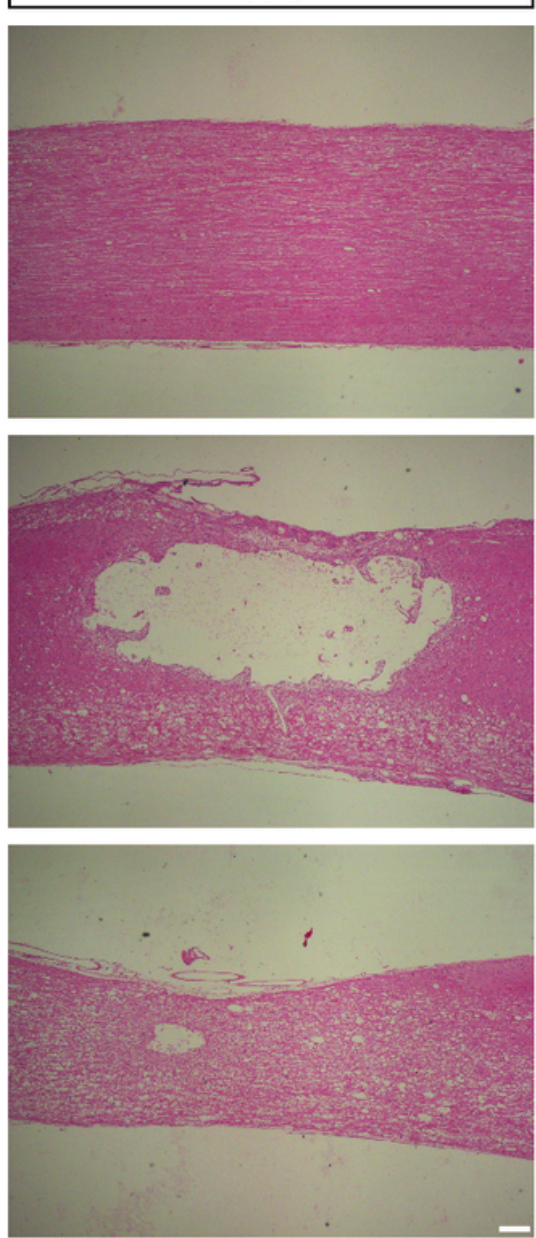

$\mathrm{B}$

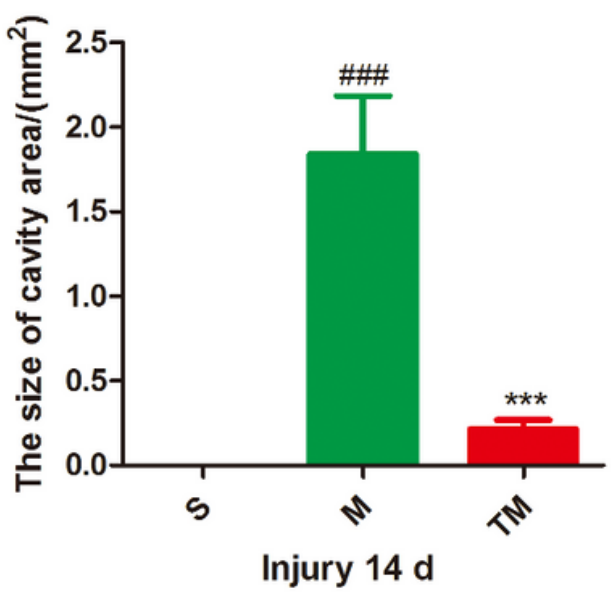

C

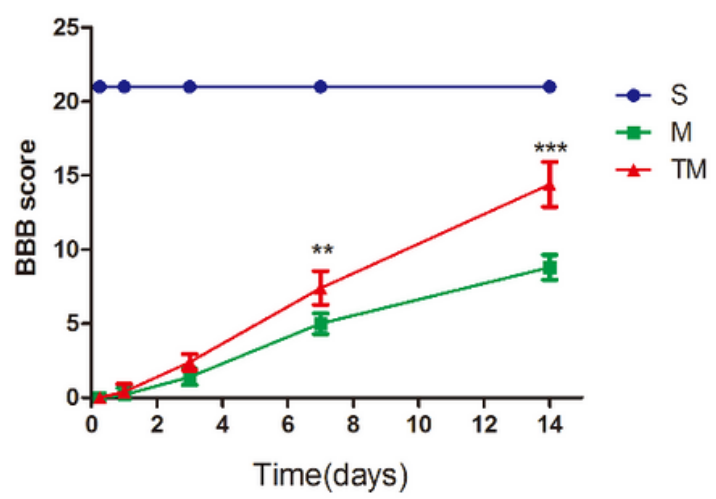

Figure 1

TT decreased tissue structure damage and improved functional recovery after SCl. A HE-stained longitudinal sections at $7 \mathrm{~d}$ and $14 \mathrm{~d}$ after SCl. Scale bar $=200 \mu \mathrm{m}$. B Quantification of the cavity area; columns represent the mean $\pm S D(n=5)$. C BBB scores in the $S, M$, and TM groups. \#p $<0.05$ as $M$ group versus $S$ group, ${ }^{\star} p<0.05$ as TM group versus $M$ group. (\#p, ${ }^{*} p<0.05 ; \# \# p,{ }^{\star \star} p<0.01 ; \# \# \# p,{ }^{\star \star *} p<0.001$ ) 

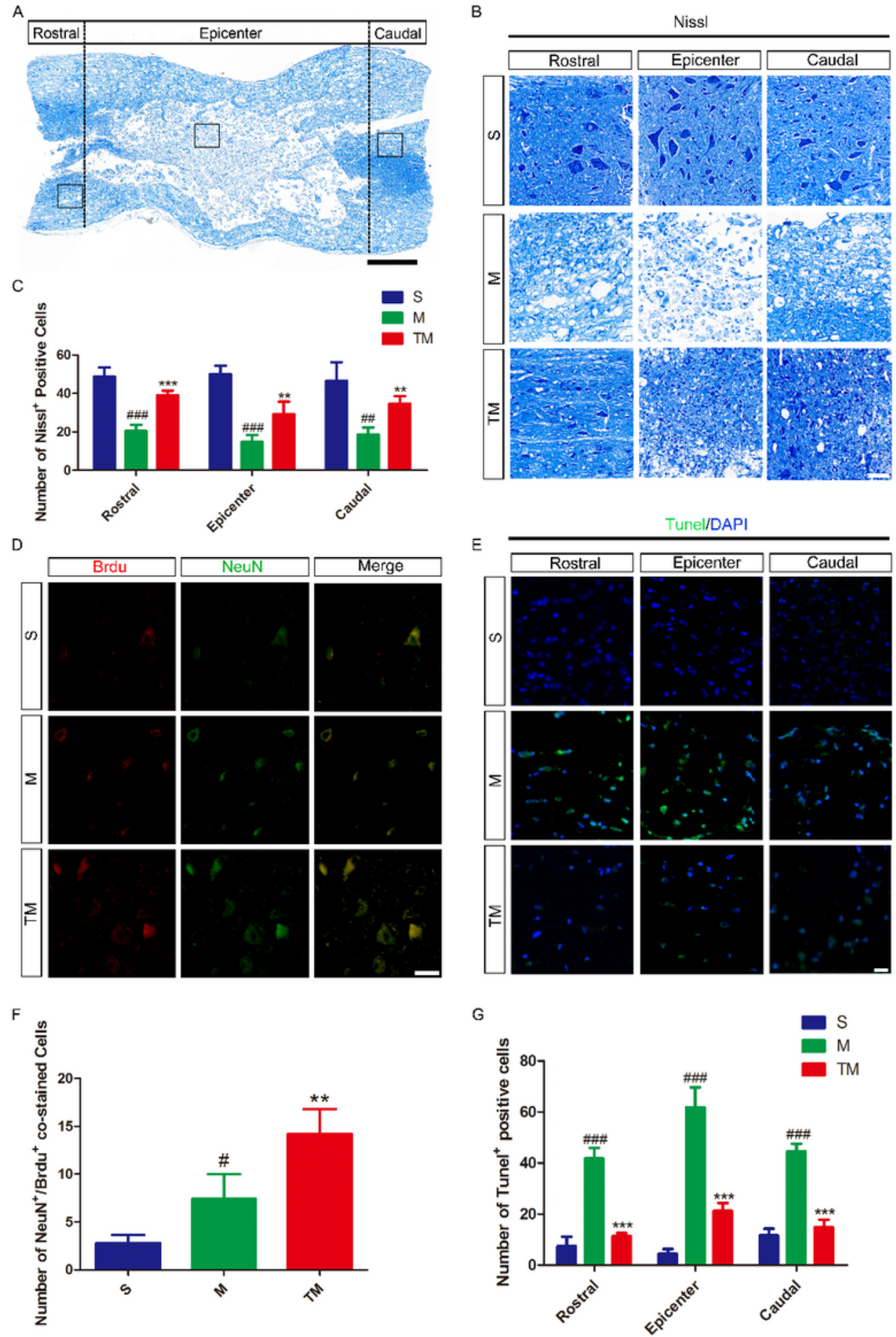

Figure 2

TT promoted the regeneration of neurons and reduced the apoptosis around the lesion. A Schematic diagram of the regions sampled after SCl. Scale bar $=500 \mu \mathrm{m}$. B Nissl staining in the S, M and TM groups from different regions at $7 \mathrm{~d}$. Scale bar $=50 \mu \mathrm{m}$. C Quantification of the Nissl+ positive cells; columns represent the mean $\pm S D(n=5)$. D Double staining at $14 \mathrm{~d}$ after $\mathrm{SCl}$ for the Brudu/NeuN. Red: Brdu; green: NeuN. Scale bar, $20 \mu \mathrm{m}$. E TUNEL staining in the rostral/epicenter/caudal at $7 \mathrm{~d}$ after SCl. 
Scale bar represent $20 \mu \mathrm{m}$. F-G Quantification data of Brdu+/NeuN+ and Tunel+ in each group, columns represent the mean $\pm S D, n=5$. (\#p, $\left.{ }^{*} p<0.05 ; \# \# p, * * p<0.01 ; \# \# \# p, * * * p<0.001\right)$
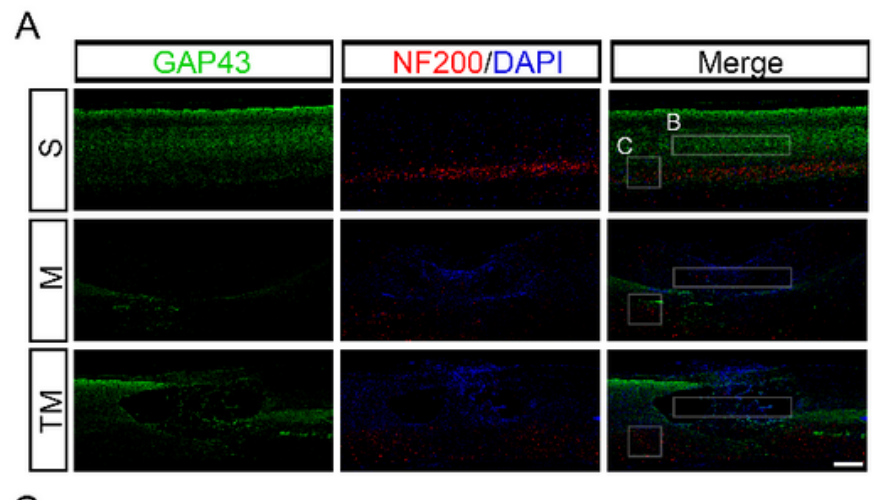

B
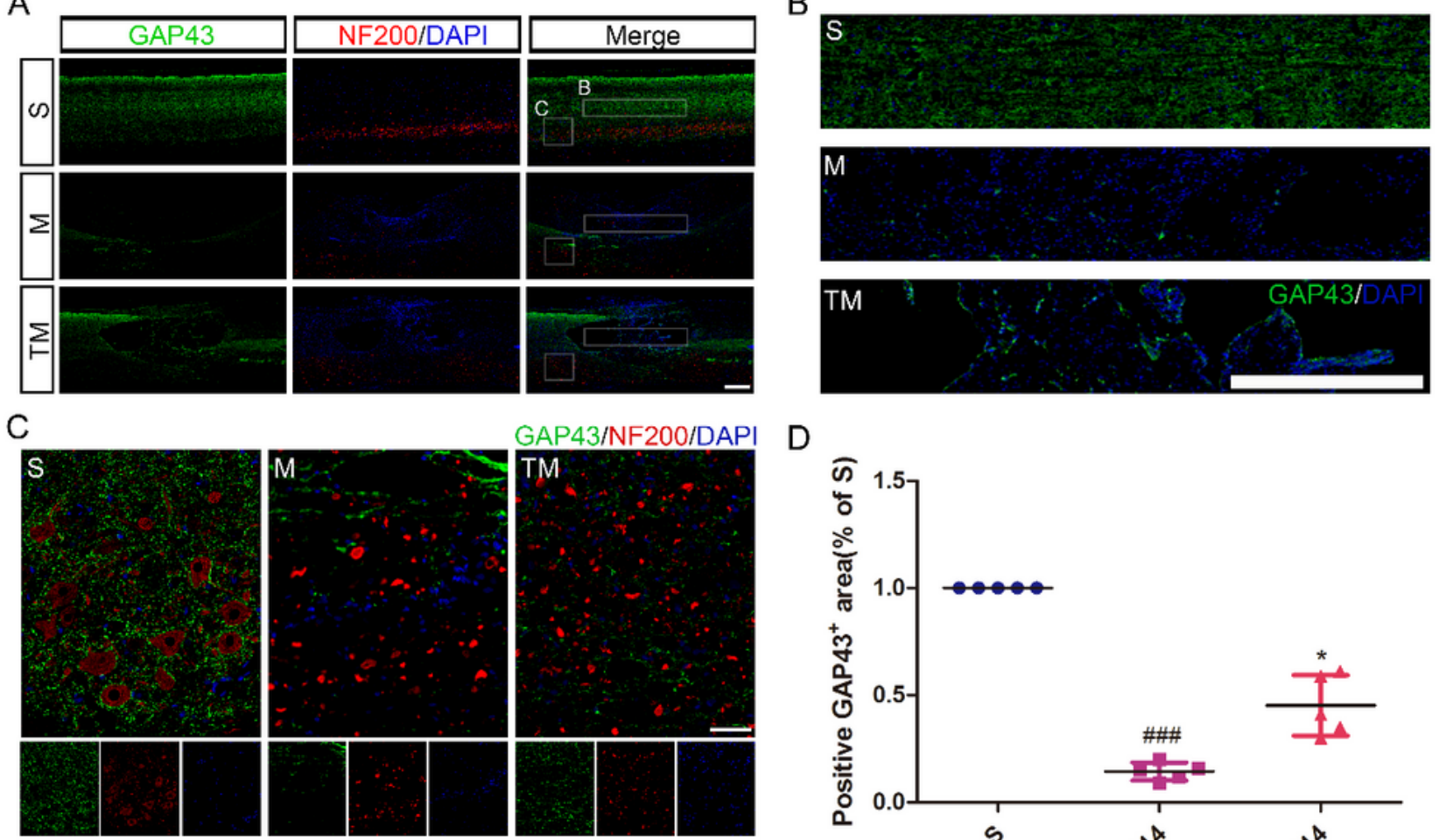

D

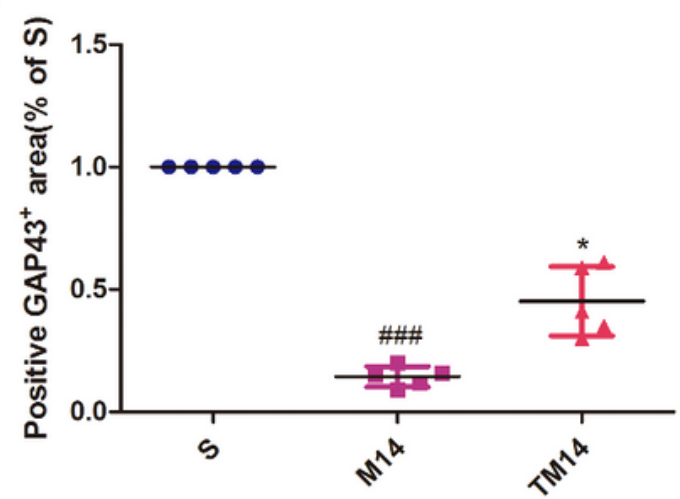

$\mathrm{E}$

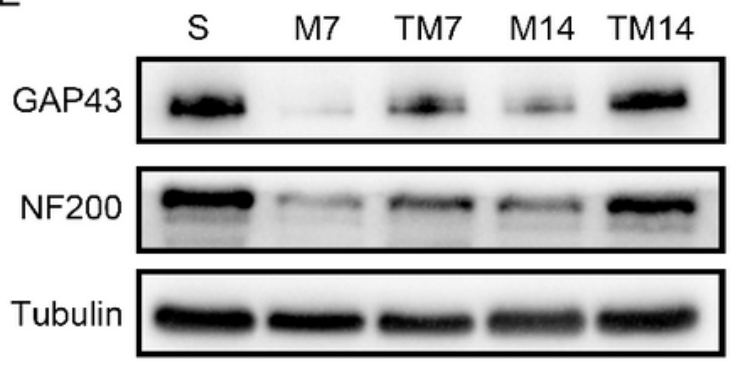

F
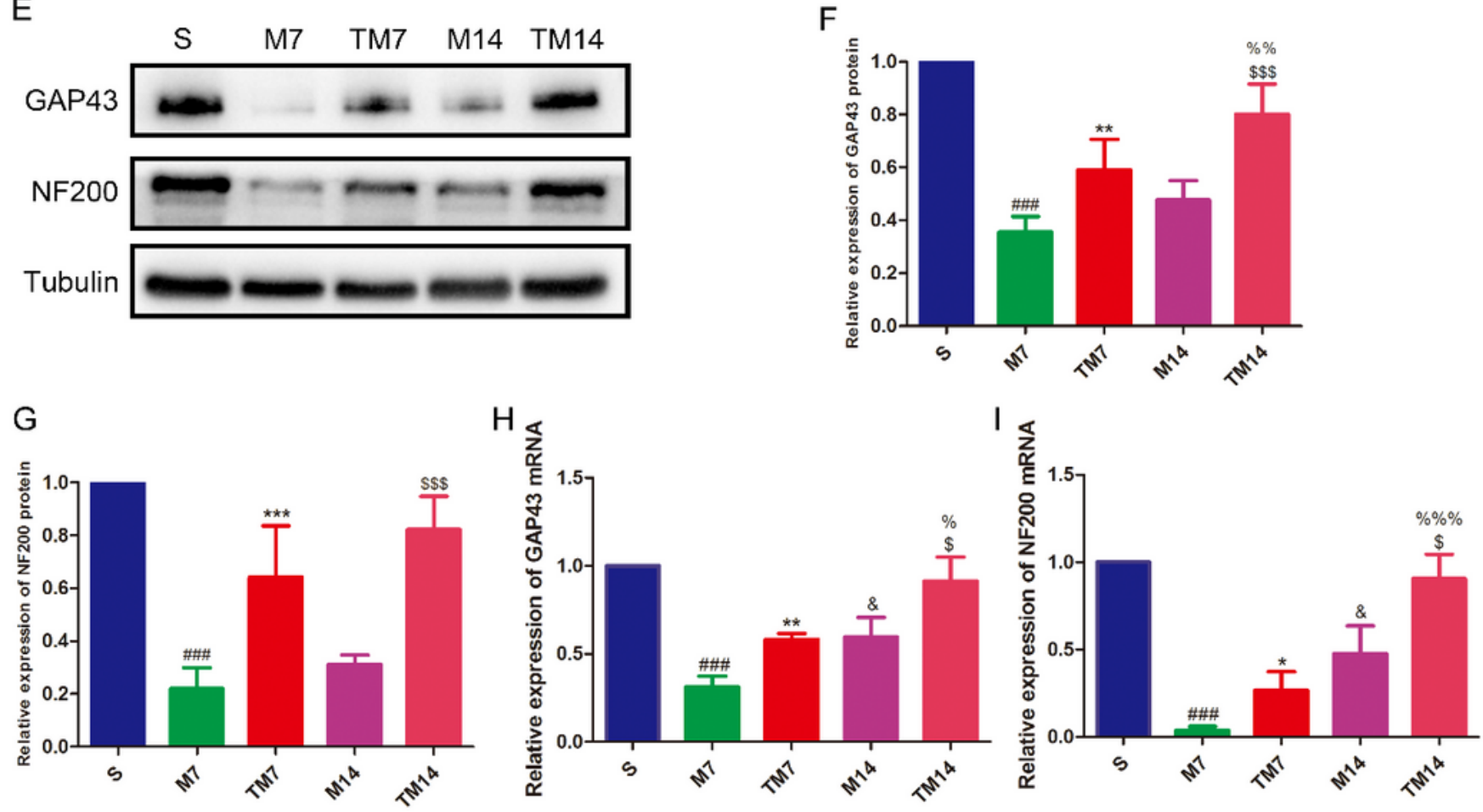

Figure 3

TT promoted axonal outgrowth after SCl. A-C Double staining of sections from the spinal cord in each group of rats for GAP43 (green)/NF200 (red)/DAPI(blue). Scale bars are $500 \mu \mathrm{m}$ (A-B) and $50 \mu \mathrm{m}(\mathrm{C})$, respectively. D Quantification of the GAP43+ cells; columns represent the mean $\pm S D(n=5)$. E Western 
blots and F-G quantification of GAP43 and NF200 proteins in each group; columns represent the mean \pm SD $(n=5)$. E-F Represent RT-qPCR analysis of GAP43 and NF200 mRNA expression at $7 d$ and $14 d$ in different groups; columns represent mean $\pm S D, n=5$. \& $<0.05$ as M14 group versus M7 group, $\$ p<$ 0.05 as TM14 group versus M14 group, \%p < 0.05 as TM14 group versus TM7 group. (\#p, *p, \&p, $\$ p, \% p<$ $0.05 ; \# \# p,{ }^{\star *} p, \& \& p$,

\section{$p$,}

$\$ p, \% \% p<0.001)$ 


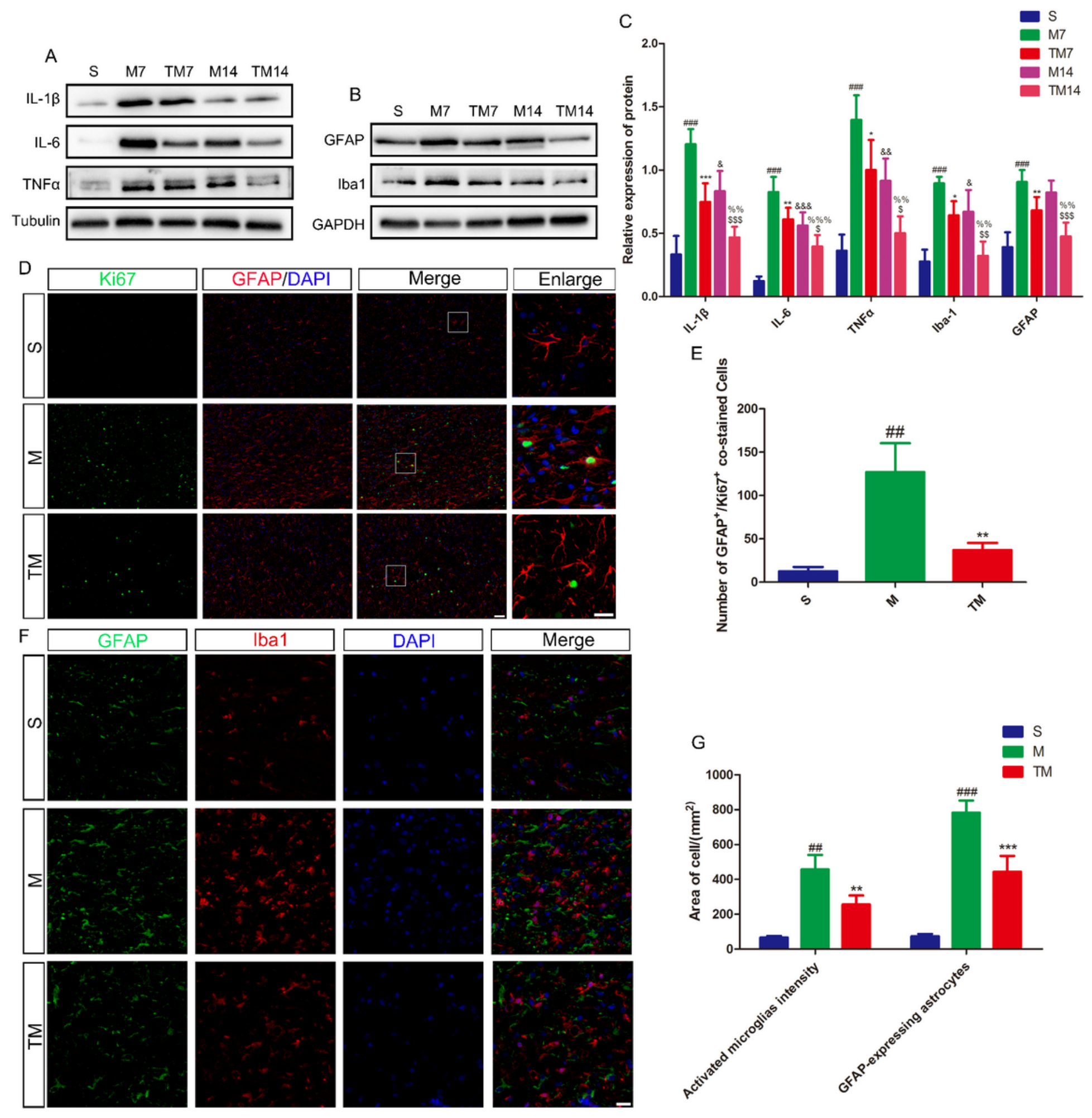

Figure 4

TT inhibited the inflammatory activation of microglia and astrocyte. A-B Western blots and C quantification of IL-4, IL-6, TNFa, GFAP and Iba1 proteins in each group after SCl; columns represent the mean $\pm S D(n=5)$. D Double staining (green: Ki67; red: GFAP; blue: DAPI) and E quantification of $\mathrm{GFAP}+/ \mathrm{Ki} 67+$ cells; columns represent the mean $\pm \mathrm{SD}(\mathrm{n}=5)$. F Double staining (green: GFAP; red: Iba1; blue: DAPI) and G quantification of positive cells area. (\#p, *p, \&p, \$p, \%p<0.05; \#\#p, **p, \&\&p, 
$\$ p, \% \% \% p<0.001)$
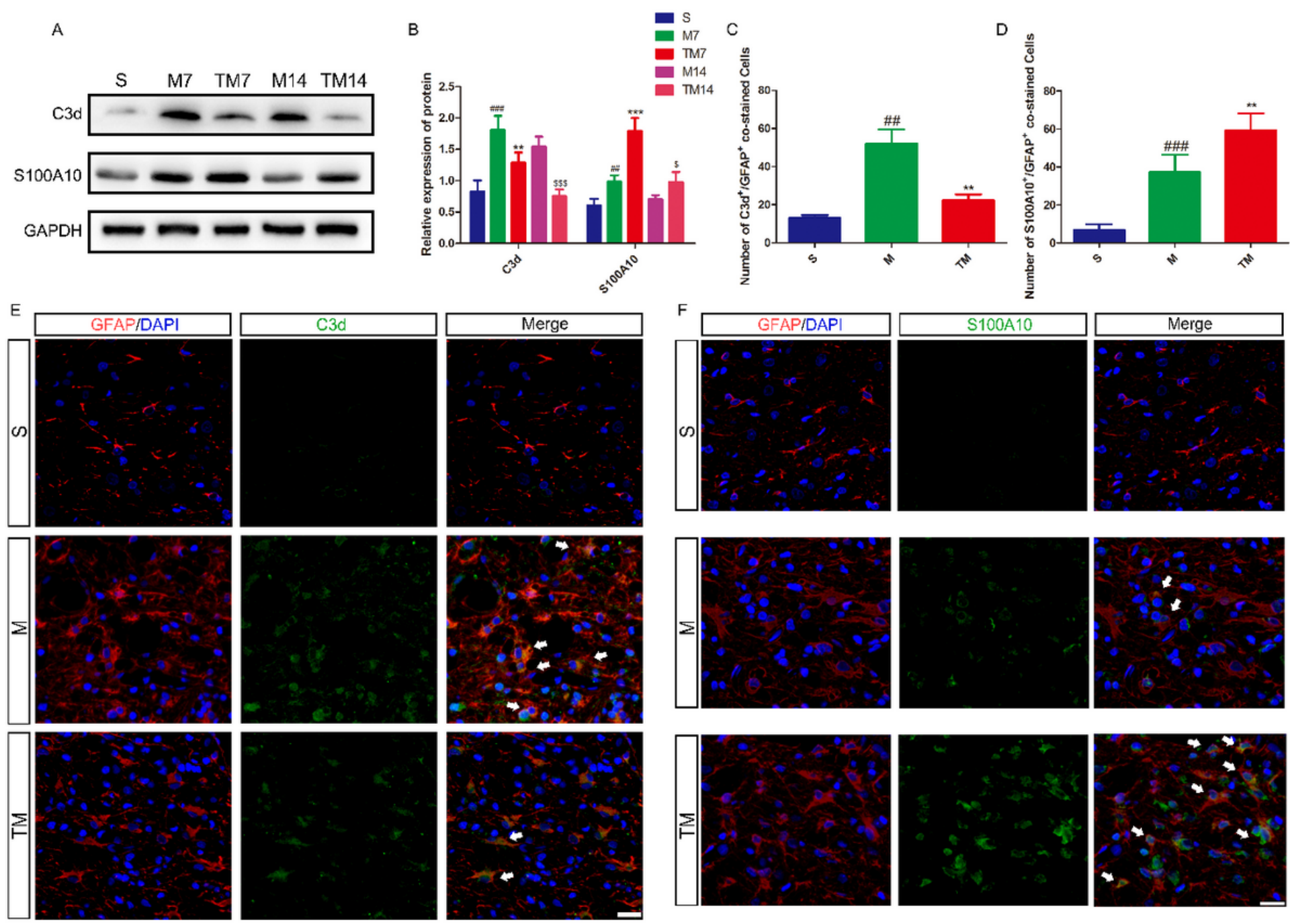

Figure 5

TT inhibited A1 type reactive astrocytes and promoted A2 type reactive astrocytes around the lesion. A Western blots and B quantification of C3d and S100A10 proteins in each group after SCl; columns represent the mean $\pm S D(n=5)$.C-D Quantification of C3d+; S100A10+/GFAP+ cells and E-F Double staining of sections in each group for C3d; S100A10 (green)/GFAP (red)/DAPI(blue). Scale bars are 200 $\mu \mathrm{m}$. White arrowheads indicate co-stained of C3d/S100A10 and GFAP. (\#p, ${ }^{*} p, \& p, \$ p, \% p<0.05 ; \# \# p,{ }^{* \star} p$, $\& \& p$,

$p$,

$\$ p, \% \% p<0.001)$ 

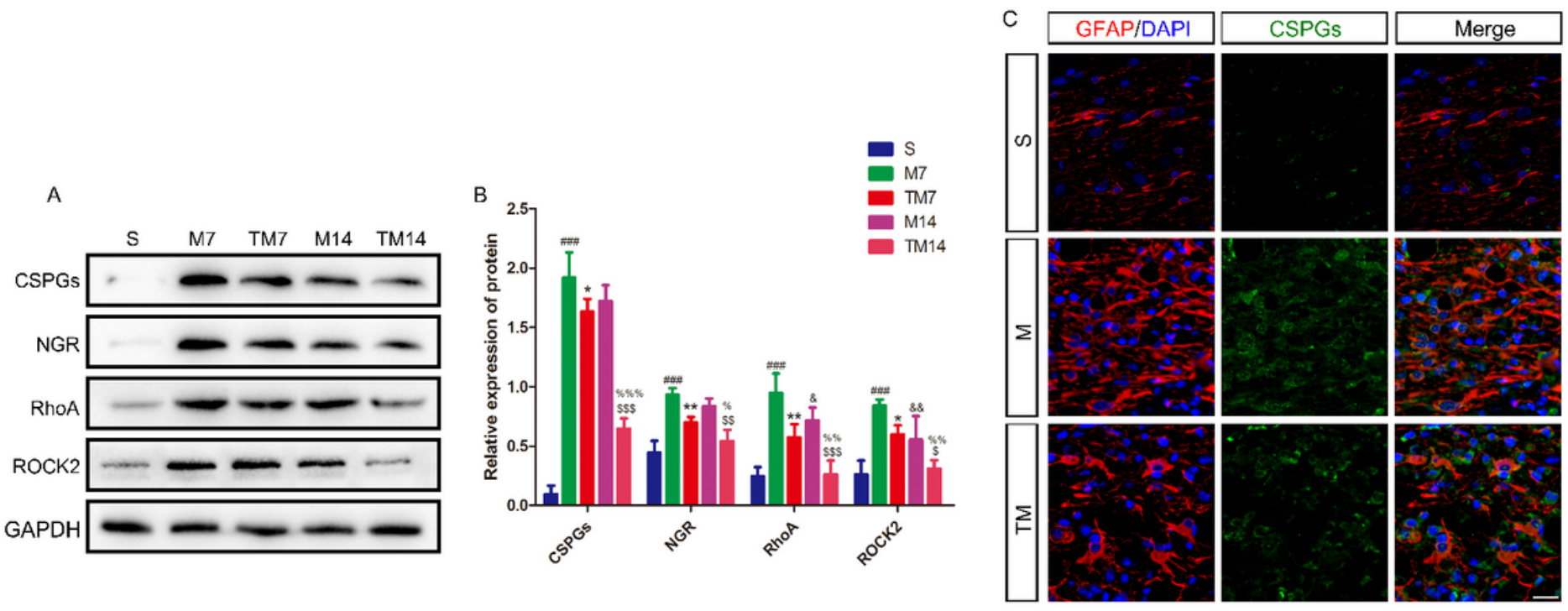

D
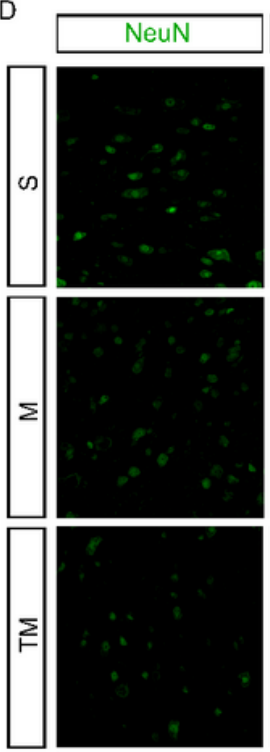

RhoA
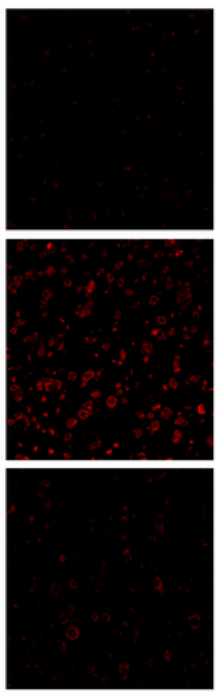

DAPI
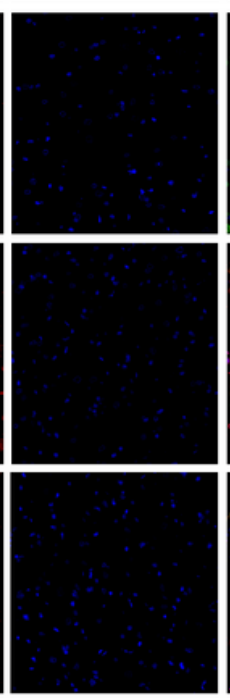

Merge
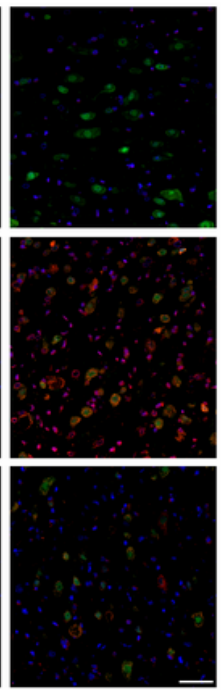

E

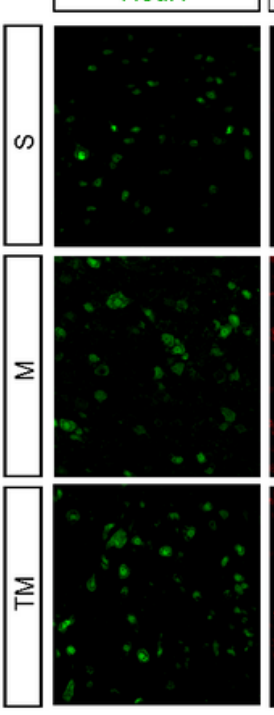

ROCK2
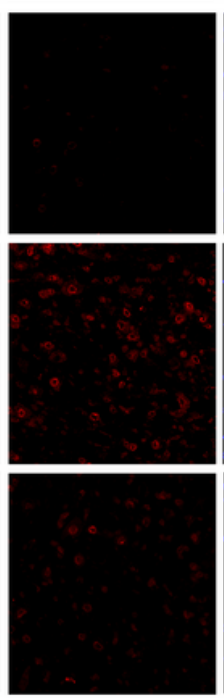

DAPI
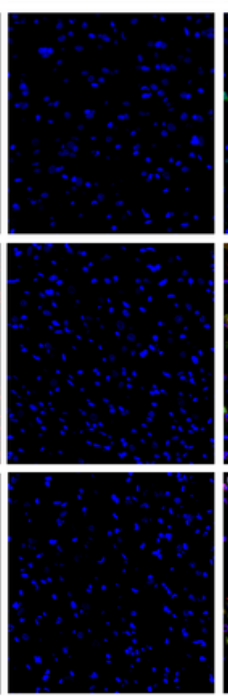

Merge

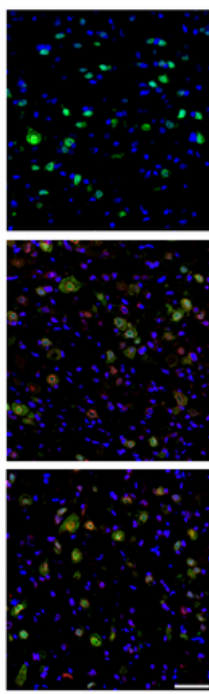

\section{Figure 6}

TT inhibited the expression of RhoA/Rock induced by astrocyte activation. A Western blots and B quantification of CSPGs, NGR, RhoA and ROCK2 proteins in each group after SCl; columns represent the mean \pm SD $(n=5)$. C Double staining for the GFAP/CSPGs/DAPI. Red: GFAP; green: CSPGs; blue:DAPI. Scale bar $=20 \mu \mathrm{m}$. D-E Double staining at $14 \mathrm{~d}$ after SCl for the RhoA; ROCK2//NeuN/DAPI. Red: RhoA/ROCK2; green: NeuN; blue:DAPI. Scale bars are $50 \mu \mathrm{m}$. (\#p, *p, \&p, \$p, \%p < 0.05; \#\#p, **p, \&\&p,

$$
p \text {, }
$$

$\$ p, \% \% \%<0.001)$ 


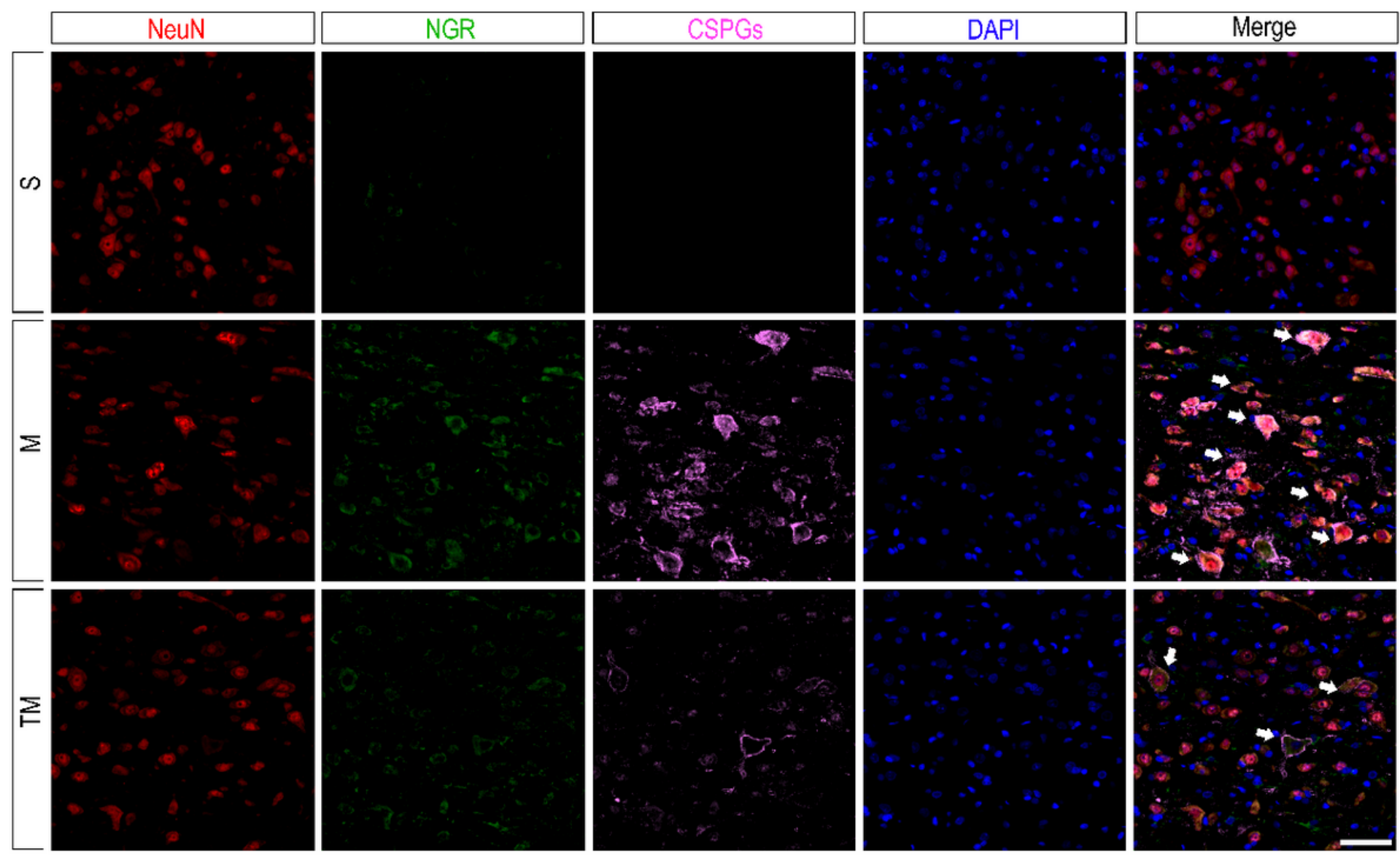

Figure 7

Localization and expression of NeuN/NGR/CSPGs/DAPI. red: NeuN; green: NGR; Pink: CSPGs; blue: DAPI. White arrowheads indicate co-stained of NeuN/NGR/CSPGs/DAPI. Scale bar, $50 \mu \mathrm{m}$. 


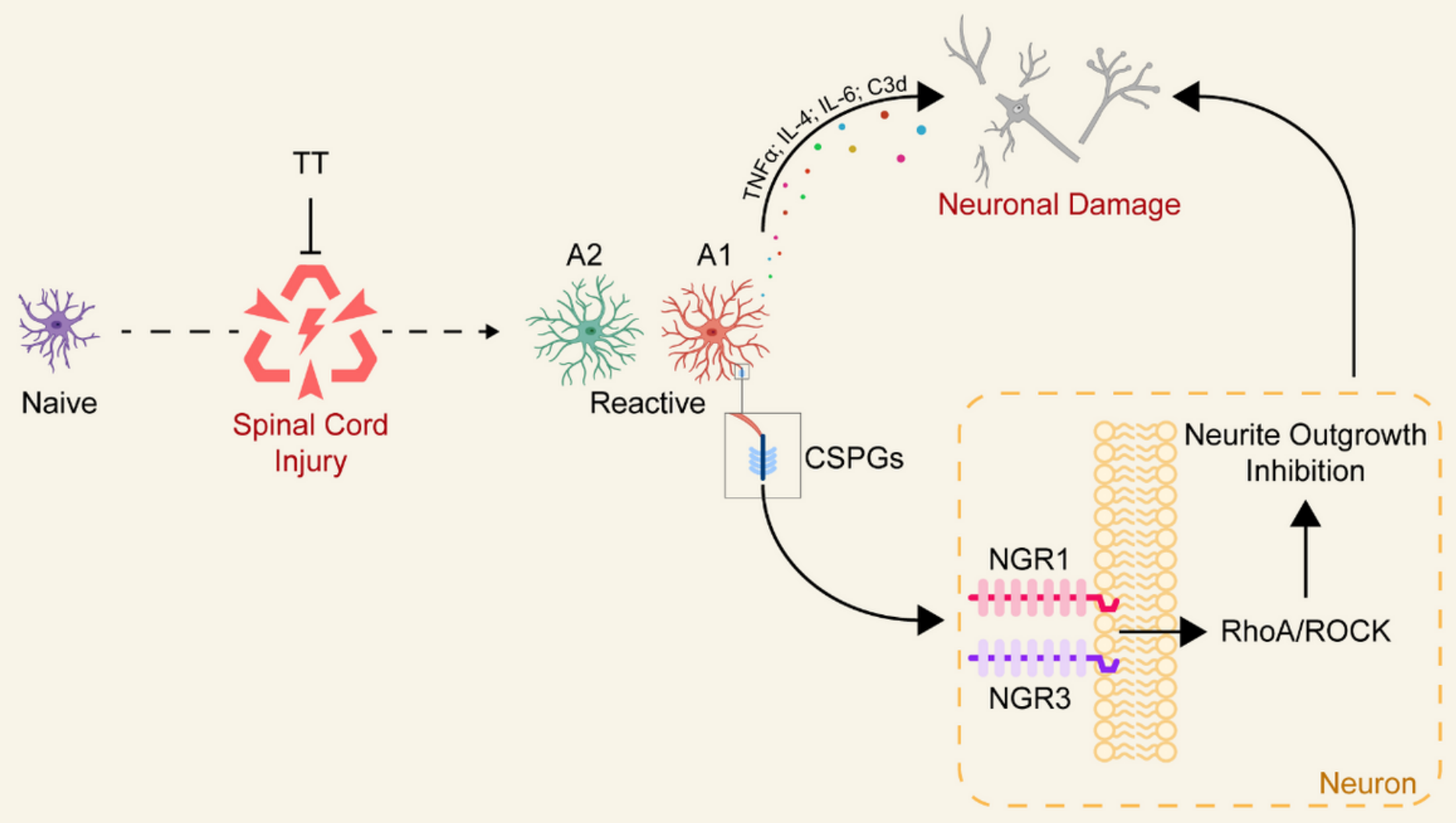

Figure 8

Proposed mechanism by which TT protects the neurons after SCl. After SCl, "Naive" astrocytes are activated, and "Reactive" astrocytes secrete inflammatory mediators to directly damage neurons. In addition, CSPGs will bind to NGR1,3, activate downstream RhoA/ROCK signalling pathway to inhibit neurite outgrowth, which indirectly damage neurons. TT can effectively inhibit the activation of astrocytes to promote axonal outgrowth after SCl. 